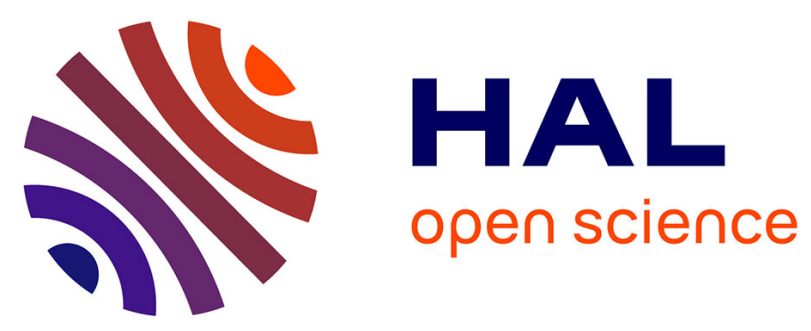

\title{
Inclined layer convection in a colloidal suspension with negative Soret coefficient at large solutal Rayleigh numbers
}

Matteo Italia, Fabrizio Croccolo, Frank Scheffold, Alberto Vaitali

\section{- To cite this version:}

Matteo Italia, Fabrizio Croccolo, Frank Scheffold, Alberto Vaitali. Inclined layer convection in a colloidal suspension with negative Soret coefficient at large solutal Rayleigh numbers. European Physical Journal E: Soft matter and biological physics, 2014, 37 (10), pp.Article number 101, Pages 1-6. 10.1140/epje/i2014-14101-7 . hal-01279144

\author{
HAL Id: hal-01279144 \\ https://hal.science/hal-01279144
}

Submitted on 24 Jan 2020

HAL is a multi-disciplinary open access archive for the deposit and dissemination of scientific research documents, whether they are published or not. The documents may come from teaching and research institutions in France or abroad, or from public or private research centers.
L'archive ouverte pluridisciplinaire HAL, est destinée au dépôt et à la diffusion de documents scientifiques de niveau recherche, publiés ou non, émanant des établissements d'enseignement et de recherche français ou étrangers, des laboratoires publics ou privés. 


\title{
Inclined layer convection in a colloidal suspension with negative Soret coefficient at large solutal Rayleigh numbers ${ }^{\star}$
}

\author{
Matteo Italia ${ }^{1}$, Fabrizio Croccolo ${ }^{2}$, Frank Scheffold $^{3}$, and Alberto Vailati ${ }^{1, a}$ \\ 1 Dipartimento di Fisica, Università degli Studi di Milano, I-20133 Milano, Italy \\ 2 Laboratoire des Fluides Complexes et leurs Réservoirs - CNRS UMR5150, Université de Pau et des Pays de l'Adour, 64600 \\ Anglet, France \\ 3 Department of Physics, University of Fribourg, CH-1700 Fribourg, Switzerland
}

Received 11 July 2014 and Received in final form 2 October 2014

Published online: 29 October 2014 - (c) EDP Sciences / Società Italiana di Fisica / Springer-Verlag 2014

\begin{abstract}
Convection in an inclined layer of fluid is affected by the presence of a component of the acceleration of gravity perpendicular to the density gradient that drives the convective motion. In this work we investigate the solutal convection of a colloidal suspension characterized by a negative Soret coefficient. Convection is induced by heating the suspension from above, and at large solutal Rayleigh numbers (of the order of $10^{7}-10^{8}$ ) convective spoke patterns form. We show that in the presence of a marginal inclination of the cell as small as $19 \mathrm{mrad}$ the isotropy of the spoke pattern is broken and the convective patterns tend to align in the direction of the inclination. At intermediate inclinations of the order of $33 \mathrm{mrad}$ ordered square patterns are obtained, while at inclination of the order of $67 \mathrm{mrad}$ the strong shear flow determined by the inclination gives rise to ascending and descending sheets of fluid aligned parallel to the direction of inclination.
\end{abstract}

\section{Introduction}

A liquid layer under the action of a temperature gradient can be either in a stable or unstable configuration, depending on the orientation of the density gradient generated by thermal dilation relative to the acceleration of gravity. The most investigated configuration is the RayleighBénard one, where the liquid is heated from below and the density gradient and the acceleration of gravity $g$ are anti-parallel [1]. The relevant parameter to characterize the stability of this system is the Rayleigh number $R a=g \Delta \rho_{T} h^{3} /(\eta \kappa)$, where $g$ is the acceleration of gravity, $\Delta \rho_{T}$ the density difference generated by thermal dilation of the liquid, $h$ the thickness of the layer, $\eta$ the shear viscosity and $\kappa$ the thermal diffusivity. Under this configuration, when $R a$ exceeds the threshold value $R a_{c}=1708$, the symmetry of the system gets broken by non-equilibrium fluctuations and convective motions develop inside the liquid. The presence of a component of $\boldsymbol{g}$ perpendicular to the density gradient determined by an inclination of the liquid layer generates a Large Scale Shear Flow that strongly affects the convective motions. For simple fluids close to the threshold of the instability, it has been shown both theoretically $[2-4]$ and experimentally [5-8] that for

\footnotetext{
* Contribution to the Topical Issue "Thermal nonequilibrium phenomena in multi-component fluids" edited by Fabrizio Croccolo and Henri Battaler.

a e-mail: alberto.vailati@unimi.it
}

inclination angles in the range $10^{\circ}-120^{\circ}$ the interplay between Rayleigh-Bénard convection and Large Scale Flow gives rise to a rich phase diagram characterized by a wide range of states, which include longitudinal rolls, cross-rolls and oscillations. At large Rayleigh numbers of the order of $10^{8}$, a region where convection becomes turbulent, a small tilting determines a slight change in the heat transferred [9]. In a recent work [10] we have shown that the solutal convection in a binary liquid mixture with positive Soret coefficient heated from below at $R a<R a_{c}$ is strongly affected by the presence of a marginal inclination as small as 6 mrad. Remarkably, in the presence of such a small inclination the stationary square patterns that characterize the planform in the absence of inclination [1114] evolve into Superhighway convective patterns where columns of ascending and descending liquid self-organize into ordered lanes drifting into opposite directions [10]. However, the analysis of the early stages of the instability shows that they are not affected by the presence of an inclination.

In this work we investigate the influence of a marginal inclination on the solutal convection of a colloidal suspension with negative Soret coefficient heated from above. In this case the density variation $\Delta \rho_{T}$ induced by the thermal dilation of the sample stabilizes the system. However, the temperature gradient induces a Soret mass flow $j=-\rho D\left(\nabla c+S_{T} c(1-c) \nabla T\right)$ in the upward direction and at steady state the presence of impermeable bound- 


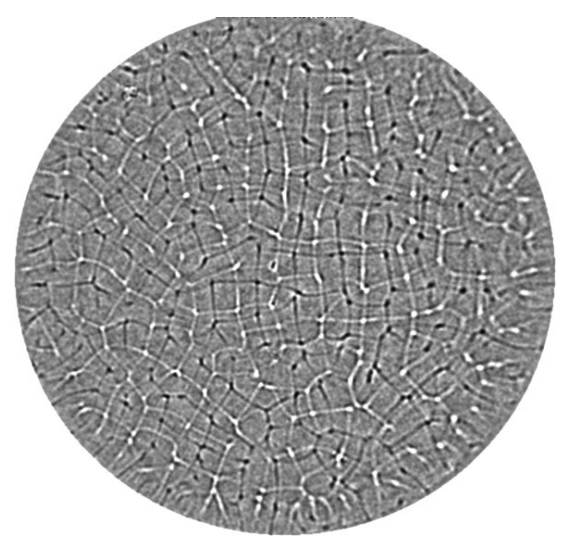

Fig. 1. Spoke pattern obtained during the solutal convection of a suspension of silica nanoparticles in water. The suspension has a negative Soret coefficient and convection is induced by heating from above at $R a_{s}=5.9 \times 10^{7}$ after $6 \times 10^{4} \mathrm{~s}$ from the onset of convection.

aries gives rise to an unstable solutal density difference across the liquid layer $\Delta \rho_{c}=\rho_{0} \beta S_{T} c(1-c) \Delta T[15]$. Here $\rho_{0}$ is the mass density of the suspension at reference temperature, $\beta$ is the solutal expansion coefficient, $c$ is the weight fraction concentration of the denser component, and $S_{T}$ is the Soret coefficient. The relative contribution of $\Delta \rho_{T}$ and $\Delta \rho_{c}$ to the overall density difference is quantified by the separation ratio $\Psi=\Delta \rho_{c} / \Delta \rho_{T}$; when $\Psi<-1$ the system is unstable when heated from above [16]. A further contribution to the destabilization of the sample comes from the fact that concentration fluctuations in colloidal suspension are characterized by decay times orders of magnitudes larger than those of temperature fluctuations. The ratio between the two time scales is expressed by the Lewis number $L e=D / \kappa$, which can be typically of the order of $10^{-4}$ for colloidal suspensions. For this reason, even a small solutal density difference can give rise to solutal convection in the presence of a stabilizing density gradient determined by the thermal dilation of the sample. The relevant parameter in this case is the solutal Rayleigh number $R a_{s}=g \Delta \rho_{c} h^{3} /(\eta D)=R a \Psi / L e$. Studies on simple fluids [17-19] and binary mixtures and colloidal suspensions [20-25] performed at large (solutal) Rayleigh numbers of the order of $10^{5}<R a_{s}<10^{9}$ have shown that the planform of the instability is a spoke pattern made by two staggered networks (fig. 1) with loops that are on average quadrilateral [25]. In the case of an instability induced by the Soret effect, the onset of the instability is accompanied by relaxation oscillations determined by the interplay between the growth of solutal boundary layers and the triggering of the convective flow $[22,23,26]$, a mechanism similar to that reported for the onset of solutal convection in suspensions of thermo-sensitive particles with positive Soret coefficient [27-29]. The main questions that we want to address with this work are: i) is there any detectable evidence of the presence of a marginal inclination during the onset of the instability in a colloidal suspension with negative Soret coefficient? ii) what is the long-term effect of the inclination on the structure of the convective planform?

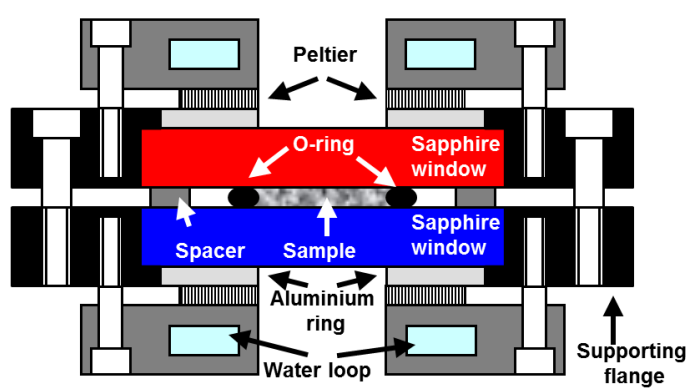

Fig. 2. Cross section of the thermal gradient cell. The heart of the cell is made by two sapphire windows that act at the same time as thermalizing plates and optical windows. Heat is injected from/to the windows by using annular Peltier elements.

\section{Materials and methods}

The sample is a colloidal suspension of silica nanoparticles with a diameter of $32 \mathrm{~nm}$ dispersed into water at a weight fraction concentration of $4.0 \%$, known commercially as LUDOX TMA. The separation ratio is $\Psi=-3.4$ and the Lewis number $L e=1.54 \times 10^{-4}$. Other thermophysical properties of the suspension are detailed in [30, 31]. In order to investigate the development of convective patterns we need to access the sample optically from the vertical direction. For this reason, the sample is sandwiched between two sapphire windows with a diameter of $70 \mathrm{~mm}$ and a thickness of $12.0 \mathrm{~mm}$. The configuration of the thermal gradient cell is similar to that adopted and tested in other experiments (fig. 2) [30-32].

The relatively high thermal conductivity of sapphire (of the order of $35 \mathrm{~W} \mathrm{~m}^{-1} \mathrm{~K}^{-1}$ ) allows to use the windows at the same time as thermalizing plates and as optical windows. The sample thickness is kept at $2.90 \mathrm{~mm}$ by means of calibrated Delrin spacers. Each sapphire window is in contact with an annular Peltier heat pump with an inner hole of diameter $27 \mathrm{~mm}$. The sample is confined laterally by an o-ring gasket with an inner diameter of $27 \mathrm{~mm}$. This feature guarantees that we are able to access optically the entire sample, even close to the lateral boundary. Each Peltier element is controlled by a Proportional-IntegralDerivative servocontrol and can transfer heat from/to a water circulation bath. The stability of the average temperature and of the temperature difference applied to the sample is of the order of $10 \mathrm{mK} / 24$ hours. The uniformity of the temperature gradient across the observation window is better than $3 \%$. The cell assembly is supported by a kinematic table accurately leveled by using a machinist level with an accuracy of $0.05 \mathrm{~mm} / \mathrm{m}$. Once levelled, the cell can be tilted by means of a calibrated screw and the inclination can be read with a resolution $60 \mu \mathrm{rad}$ by using a comparator with a resolution of $0.01 \mathrm{~mm}$. The optical technique of choice is shadowgraphy [33], a projection technique used mostly for visualization purposes in the past, which has become a highly quantitative technique during the last 15 years [34-36]. The layout of the setup is similar to that used in $[32,37]$. The light source is a superluminous diode with a wavelength of $676.5 \mathrm{~nm}, \mathrm{FWHM}$ of $14.1 \mathrm{~nm}$ and a maximum intensity of $1 \mathrm{~mW}$, coupled to a 
monomode optical fiber. The divergent beam coming out of the fiber is collimated by an achromatic doublet with a focal length of $50 \mathrm{~cm}$. The large focal length of the doublet allows to achieve a uniform illumination of the sample. The collimated beam crosses the sample in vertical direction and an achromatic doublet of focal length $10 \mathrm{~cm}$ images a plane at a distance $z=50 \mathrm{~mm}$ from the cell onto a Charged Coupled Device (CCD) detector with a resolution of $768 \times 576$ pixels. The signal coming from the CCD is sampled by a frame grabber and recorded digitally.

A typical measurement sequence (fig. 3) involves the sudden imposition of a temperature difference to the sample by heating from above. Following a latency phase, convective columns appear and gradually evolve into a spoke pattern configuration. The strength of the convective flow can be characterized by determining the contrast of each image $C(t)=\left\langle\left[I(\boldsymbol{x}, t)-I(\boldsymbol{x}, t)_{o}\right]^{2}\right\rangle_{\boldsymbol{x}}$ where $\langle\ldots\rangle_{\boldsymbol{x}}$ represents the average over the pixels of an image, and $I\left(\boldsymbol{x}, t_{o}\right)$ is a background image at time $t=0$ before applying the temperature gradient.

\section{Results and discussion}

\subsection{Relaxation oscillations at the onset of the instability}

The contrast signal remains stable for a latency time of a few hundred seconds following the imposition of the temperature gradient. This is apparent from fig. 4, which shows curves of the contrast as a function of time at different solutal Rayleigh numbers at an inclination angle of 19 mrad.

The onset of the instability is characterized by the development of solutal boundary layers located at the impermeable boundaries [24]. Due to the solutal mass flow generated by the Soret effect, colloidal particles tend to accumulate near the top boundary and to be removed from a region near the bottom boundary. The thickness $d$ of the boundary layers grows diffusively $d \propto \sqrt{D t}$ until it reaches a critical thickness $d^{*}$ when it becomes unstable $[38,24$, 28]. At the critical thickness the non-equilibrium fluctuations generated by velocity fluctuations at the boundary layer get amplified by the density gradient and they give rise to convective flows in the form of ascending and descending regions of liquid [39]. The convective flow partially destroys the boundary layers that generated it, and this gives rise to the relaxation oscillations shown in fig. $4[40,41,24]$. Theoretical models $[42-44,26]$ and experiments $[23,24]$ have shown that in the absence of inclination the critical time needed for the system to become unstable exhibits a power law dependence $\tau^{*}=25.3 R a_{s}^{-0.52}$ from the solutal Rayleigh number (circles in fig. 5). Here $\tau^{*}=t^{*} / t_{D}$ is the critical time $t^{*}$ made dimensionless by dividing it by the diffusive time across the cell height $t_{D}=h^{2} / D$.

Similar results were found for the onset of convection in suspensions of thermo-sensitive particles [27,28]. In order to assess the influence of the inclination of the sample on the time $\tau^{*}$, we performed a series of systematic
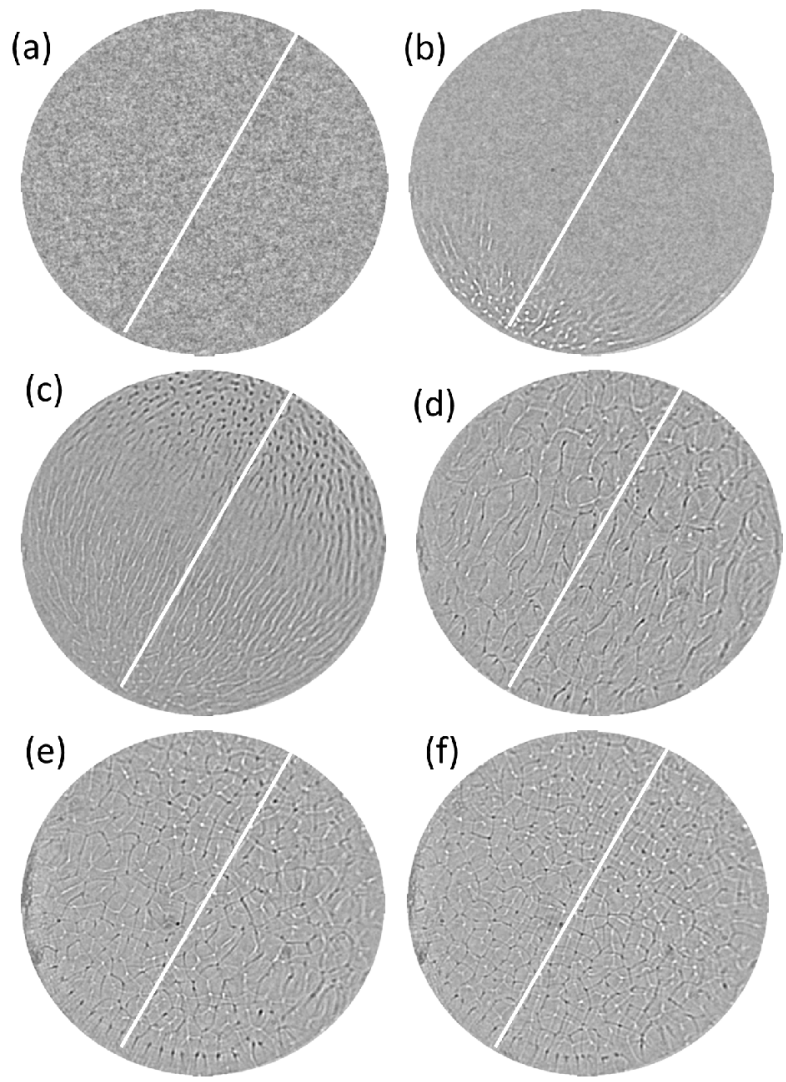

Fig. 3. Time evolution of the convective pattern at $R a_{s}=$ $1.29 \times 10^{8}$ and $\theta=33 \mathrm{mrad}$. The solid line represents the direction of the inclination. The cell is higher at the bottom left of each panel. Dark features represent regions of upwelling flow of water-rich liquid, while light features regions of downwelling flow of colloid-rich fluid. (a) At time $t=0$, when the gradient is turned on, the pattern is featureless. (b) After $t=373 \mathrm{~s}$ downwelling columns of liquid appear at the bottom left. (c) At $t=508 \mathrm{~s}$ the pattern is divided into nearly symmetric halves: upwelling column of liquid at the top right of the panel and downwelling columns at the bottom left. (d) After $1130 \mathrm{~s}$ the pattern has developed two staggered networks of ascending and descending liquid. (e) At $t=20744 \mathrm{~s}$ the networks have started the transition to a spoke pattern. (f) At $t=61617 \mathrm{~s}$ the pattern has become a fully developed spoke pattern made mostly of quadrilateral cells aligned in the direction of inclination.

measurements with solutal Rayleigh numbers in the range $1.37 \times 10^{7}<R a_{s}<1.29 \times 10^{8}$ and inclination angles $0<\theta<67 \mathrm{mrad}$. The results are shown by the squares in fig. 5. One can appreciate that the data obtained in the presence and in the absence of inclination nicely collapse onto the same curve. From the latency time we are able to determine the thickness $\delta=\sqrt{\pi \tau}$ of the boundary layers at the onset of the instability normalized by the sample thickness [24]. At the large solutal Rayleigh numbers used in this work the thickness of the boundary layers is always a small fraction of the thickness of the sample.

Although the growth dynamics of the boundary layers does not appear to be affected by the inclination, a close inspection of the convective patterns at the onset 


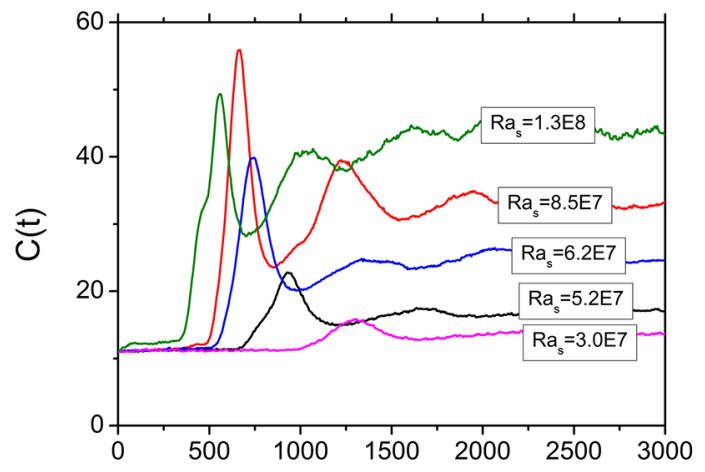

$\mathrm{t}(\mathrm{s})$

Fig. 4. Time evolution of contrast at various solutal Rayleigh numbers for an inclination of $19 \mathrm{mrad}$ with respect to the horizontal direction.

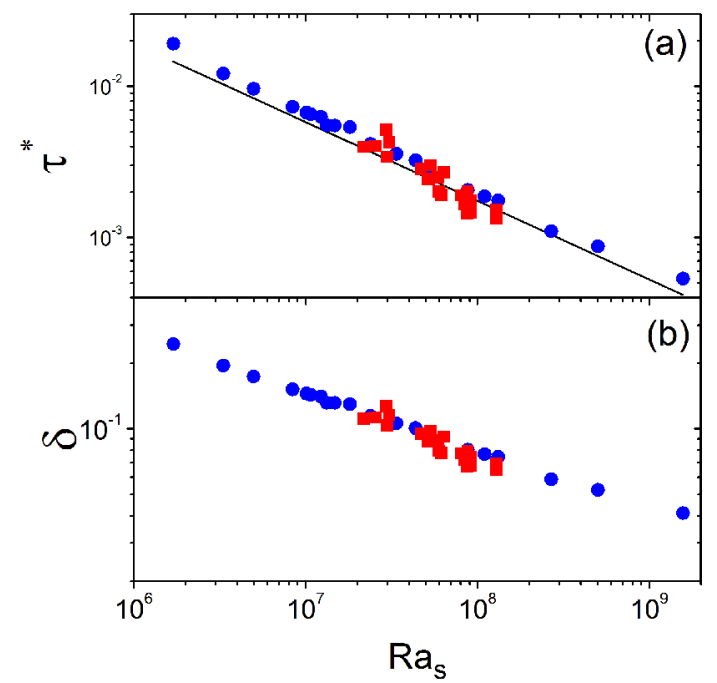

Fig. 5. (a) Latency time as a function of solutal Rayleigh number. (b) Boundary layer thickness as a function of solutal Rayleigh number. Circles correspond to results in the absence of inclination [24], while squares to results with inclination angle in the range $19 \mathrm{mrad}<\theta<67 \mathrm{mrad}$. The solid line represents the power law behavior predicted by the theory [42-44, $26]$.
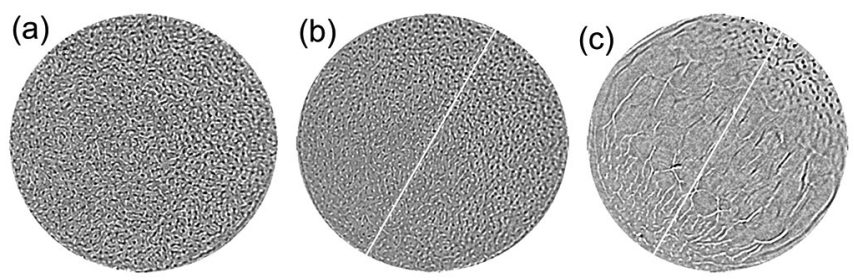

Fig. 6. Effect of the inclination of the patterns at the onset of the instability. Patterns correspond to the first peak of the contrast, when the convective flow is stronger. The solid line represents the direction of the inclination. (a) Isotropic pattern at $R a_{s}=5.9 \times 10^{7}$ and $\theta=0 \mathrm{mrad}$. (b) At $R a_{s}=1.3 \times 10^{8}$ and $\theta=19 \mathrm{mrad}$ upwelling and downwelling flows tend to develop into opposite halves of the cell. (c) Shear pattern at $R a_{s}=$ $8.1 \times 10^{7}$ and $\theta=67 \mathrm{mrad}$. of the instability reveals that they are influenced by the inclination from the beginning of the instability (fig. 6). Quite generally, the start of the growth of the contrast corresponds to the appearance of white structures associated to the downwelling columnar flow of colloid-rich liquid (fig. 3b). This temporal asymmetry between the appearance of black and white structures occurs independently of the presence of an inclination, while the inclination gives rise to an additional spatial asymmetry. In the absence of inclination, when the contrast reaches its peak the pattern is characterized by a uniform distribution of ascending and descending structures (fig. 6(a)). For intermediate inclinations of $33 \mathrm{mrad}$ white downwelling structures dominate the highest side (bottom left of fig. 6(b)) of the cell and black upwelling structures the lowest side (top right of fig. 6(b)). At larger inclinations of the order of $67 \mathrm{mrad}$ the central part of the cell is dominated by ascending and descending sheets of liquid, and the columnar flow is confined in regions close to the highest and lowest side of the cell (fig. 6(c)).

\subsection{Long-term behavior}

At large times the convective pattern arranges into a spoke pattern configuration for inclination angles smaller than $66 \mathrm{mrad}$. Spoke patterns are made by two staggered polygonal networks [17-19] (fig. 1). Each node of the network represents an ascending/descending column of liquid and is surrounded on average by four descending/ascending columns [25]. Spoke patterns are generated during convection in simple fluid and binary mixtures near the transition to turbulent convection, when the Rayleigh number, or solutal Rayleigh number, is of the order of $10^{5}$ or larger. The patterns are isotropic and any orientation imposed by the lateral boundaries becomes irrelevant at a distance from them comparable to the thickness of the cell, provided that the aspect ratio of the cell is large enough. The inclination of the cell determines the presence of a component of the gravity force perpendicular to the density gradient, which gives rise to a shear motion of the boundary layers in the direction of the inclination (fig. 7). The top boundary layer, rich in colloidal particles, slides down along the top sapphire window, while the bottom boundary layer slides up along the bottom window. As a result, the vertical columns of ascending and descending liquid characteristic of the spoke pattern configuration are dragged by the boundary layers that originated them and tend to drift into opposite directions.

Figure 8 shows some configurations obtained at late times larger than $6 \times 10^{4} \mathrm{~s}$ under various inclination conditions. The first panel (fig. 8(a)) corresponds to the base state in the absence of inclination. Under these conditions the pattern is a spoke pattern. From its power spectrum (fig. 8(b)) one can appreciate that the pattern is nearly isotropic. Panel 8(c) shows the pattern obtained in the presence of an inclination of $19 \mathrm{mrad}$. Near the lateral boundaries of the cell the geometry of the pattern is constrained by the boundary conditions. However, in the central part of the cell the boundary conditions become ir- 


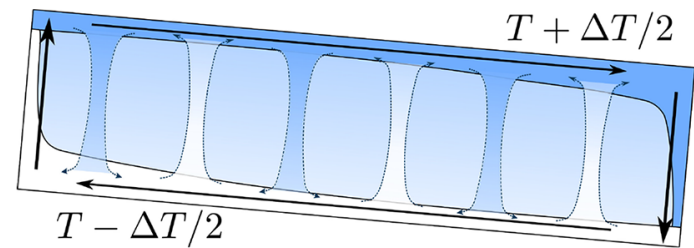

Fig. 7. Large-scale shear flow. The columnar solutal flow in the central part of the cell is accompanied by a shear motion of the boundary layers into opposite directions. The top boundary layer is denser than the liquid below it and tends to slide down along the top sapphire window. The bottom boundary layer is lighter than the fluid above it and tends to slide up along the bottom sapphire window.

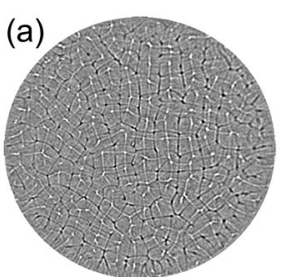

(b)

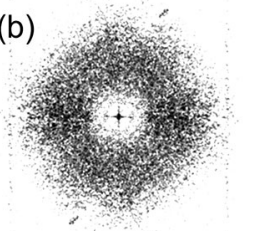

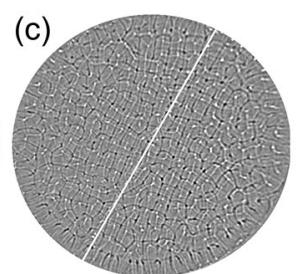

(d)

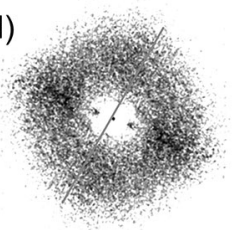

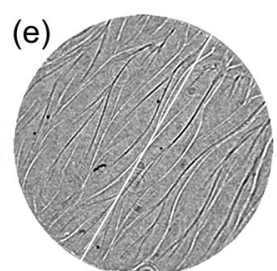

(f)

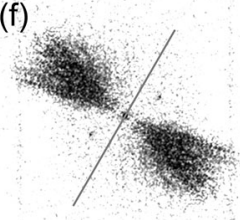

Fig. 8. Long-term convective patterns and power spectra. The solid lines correspond to the direction of inclination. (a) Spoke pattern obtained in the absence of inclination at $R a_{s}=5.9 \times 10^{7}$. (b) Isotropic power spectrum of (a). (c) Pattern obtained at $\theta=19 \mathrm{mrad}$ and $R a_{s}=1.3 \times 10^{8}$. Cells at the center of the pattern are on average quadrilateral and aligned in the direction of the inclination. This is reflected in the power spectrum in (d). (e) Shear pattern obtained at $\theta=67 \mathrm{mrad}$ and $R a_{s}=8.1 \times 10^{7}$. The spectrum in (f) shows the presence of ondulated rolls, mostly parallel to the direction of inclination.

relevant and one can appreciate that the spoke patterns rearrange into square cells aligned approximately parallel to the direction of inclination. The presence of a square pattern is apparent in the power spectrum of the pattern (fig. 8(d)), which shows the presence of two characteristic wave vectors aligned parallel and perpendicular to the direction of inclination. At larger inclinations of the order of $67 \mathrm{mrad}$ the large-scale shear flow becomes stronger and the columnar flow disappears. Solutal convection takes the form of ascending and descending sheets aligned approximately parallel to the direction of inclination (fig. 8(e)). The pattern becomes strongly anisotropic, as witnessed by the power spectrum, which develops two wings perpendicular to the direction of inclination (fig. 8(f)). Under this condition the pattern is very similar to those obtained for simple fluid in the presence of shear induced by a movement of the horizontal boundaries into opposite directions [45].

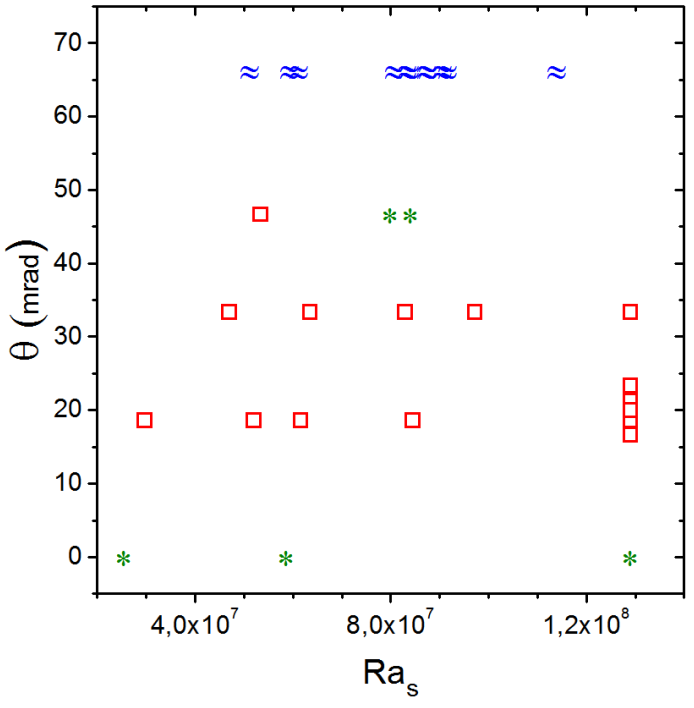

Fig. 9. Phase diagram of long-term convective behavior. Stars: spoke patterns; squares: square patterns; waves: ondulated rolls.

By performing systematic measurements at various solutal Rayleigh numbers and inclinations we were able to obtain a phase diagram for the long-term behavior of the convective patterns (fig. 9). Quite generally, in the absence of inclination the pattern is a spoke pattern (stars in fig. 9). In the presence of small inclinations, the solutal columnar flow is efficient in dissipating the excess of mass at the boundary layers, and the effect of the inclination is limited to a rearrangement of the position of the columns located at the vertexes of the spoke pattern; this gives rise to two staggered square patterns aligned parallel to the inclination (squares in fig. 9). Under this condition each ascending (descending) column is caged by four descending (ascending) columns and this gives rise to a jamming of the pattern that prevents further motion in the direction of inclination. At larger inclinations the caging mechanism is not effective any more and the convective flow is dominated by the large-scale flow, which favors the rearrangement of aligned columns into ondulated rolls (waves in fig. 9).

\section{Conclusions}

Convection in a suspension of silica colloidal particles in water with negative Soret coefficient gives rise to spoke patterns when the system is heated from above at solutal Rayleigh numbers larger than $10^{5}$. The presence of a marginal inclination as small as 19 mrad strongly affects the pattern formation. The overall growth of solutal boundary layers preceding the onset of convection is not affected much. However, the inclination affects even the early stages of the instability and gives rise to a nonisotropic destabilization of the boundary layers. At large times, the interplay between solutal convection and largescale shear flow gives rise to an orientation of the patterns at inclinations smaller than about $50 \mathrm{mrad}$ and to the development of ondulated rolls at inclinations of $67 \mathrm{mrad}$. 
AV acknowledges discussion with Roberto Cerbino, Fabio Giavazzi and Stefano Mazzoni. We thank Grace Davison for the supply of the LUDOX sample. Work supported by the Swiss National Science Foundation (projects 132736 and 149867).

\section{References}

1. M.C. Cross, P.C. Hohenberg, Rev. Mod. Phys. 65, 851 (1993).

2. R.M. Clever, F.H. Busse, J. Fluid. Mech. 81, 107 (1977).

3. F.H. Busse, R.M. Clever, J. Eng. Math. 26, 1 (1992).

4. F.H. Busse, R.M. Clever, Phys. Fluids 12, 2137 (2000).

5. K.E. Daniels, B.B. Plapp, E. Bodenschatz, Phys. Rev. Lett. 84, 5320 (2000).

6. K.E. Daniels, E. Bodenschatz, Phys. Rev. Lett. 88, 034501 (2002).

7. G. Seiden, S. Weiss, J.H. McCoy, W. Pesch, E. Bodenschatz, Phys. Rev. Lett. 101, 214503 (2008).

8. S. Weiss, G. Seiden, E. Bodenschatz, New J. Phys. 14, 053010 (2012).

9. S. Weiss, G. Ahlers, J. Fluid Mech. 715, 314 (2013).

10. F. Croccolo, F. Scheffold, A. Vailati, Phys. Rev. Lett. 111, 114502 (2013).

11. P. Le Gal, A. Pocheau, V. Croquette, Phys. Rev. Lett. 54, 2501 (1985).

12. E. Moses, V. Steinberg, Phys. Rev. Lett. 57, 2018 (1986).

13. Ch. Jung, B. Huke, M. Lücke, Phys. Rev. Lett. 81, 3651 (1998).

14. S. Weggler, B. Huke, M. Lücke, Phys. Rev. E 81, 016309 (2010).

15. S.R. de Groot, P. Mazur, Nonequilibrium thermodynamics (North-Holland, Amsterdam, 1962).

16. J.K. Platten, J.C. Legros, Convection in Liquids (Springer, Berlin, 1984).

17. F.H. Busse, J.A. Whitehead, J. Fluid Mech. 66, 67 (1974).

18. J.A. Whitehead, B. Parsons, Geophys. Astrophys. Fluid Dyn. 9, 201 (1978).

19. F.H. Busse, Acta Mech. 4, 11 (1994).

20. A. La Porta, C.M. Surko, Phys. Rev. Lett. 80, 3759 (1998).
21. R. Cerbino, A. Vailati, M. Giglio, Phys. Rev. E 66, 055301 (2002).

22. R. Cerbino, A. Vailati, M. Giglio, Philos. Mag. 83, 2023 (2003).

23. S. Mazzoni, R. Cerbino, D. Brogioli, A. Vailati, M. Giglio, Eur. Phys. J. E 15, 305 (2004).

24. R. Cerbino, S. Mazzoni, A. Vailati, M. Giglio, Phys. Rev. Lett. 94, 064501 (2005).

25. S. Mazzoni, F. Giavazzi, R. Cerbino, M. Giglio, A. Vailati, Phys. Rev. Lett. 100, 188104 (2008).

26. M.C. Kim, Korean. J. Chem. Eng. 30, 831 (2013).

27. F. Winkel, S. Messlinger, W. Schöpf, I. Rehberg, M. Siebenbürger, M. Ballauff, New J. Phys 12, 053003 (2010).

28. S. Messlinger, C. Kramer, J.J. Schmied, F. Winkel, W. Schöpf, I. Rehberg, Phys. Rev. E 88, 053019 (2013).

29. S. Messlinger, W. Schöpf, I. Rehberg, Int. J. Heat Mass Transfer 62, 336 (2013).

30. G. Donzelli, R. Cerbino, A. Vailati, Phys. Rev. Lett. 102, 104503 (2009).

31. M. Bernardin, F. Comitani, A. Vailati, Phys. Rev. E 85, 066321 (2012).

32. A. Vailati, R. Cerbino, S. Mazzoni, C.J. Takacs, D.S. Cannell, M. Giglio, Nat. Commun. 2, 290 (2011).

33. G.S. Settles, Schlieren and Shadowgraph Techniques (Springer, Berlin, 2001).

34. S. Trainoff, D.S. Cannell, Phys. Fluids 14, 1340 (2002).

35. R. Cerbino, A. Vailati, Curr. Opin. Colloid Interface Sci. 14, $416(2009)$.

36. F. Croccolo, D. Brogioli, Appl. Opt. 50, 3419 (2011)

37. F. Croccolo, H. Bataller, F. Scheffold, J. Chem. Phys. 137, $234202(2012)$.

38. L.N. Howard, Proceedings of the Eleventh International Congress of Applied Mechanics, Munich, 1964 (Springer, Berlin, 1966) pp. 1109-1115.

39. F. Giavazzi, A. Vailati, Phys. Rev. E 80, 015303 (2009).

40. V. Degiorgio, Phys. Rev. Lett. 41, 1293 (1978).

41. A. Ryskin, H. Pleiner, Phys. Rev. E 71, 056303 (2005).

42. M.C. Kim, C.K. Choi, Phys. Rev. E 76, 036302 (2007).

43. M.C. Kim, C.K. Choi, J.-K. Yeo, Phys. Fluids 19, 084103 (2007).

44. M.C. Kim, Eur. Phys. J. E 34, 27 (2011).

45. D.P. McKenzie, F. Richter, Sci. Am. 235, 72 (1976). 